\title{
The Impact of Initial Systemic Inflammatory Response After Aneurysmal Subarachnoid Hemorrhage
}

\author{
Weiying ZHONG ${ }^{1}$, Zhiyong ZHANG ${ }^{2}$, Peng ZHAO ${ }^{1}$, Jie SHEN ${ }^{1}$, Xueen LI $^{1}$, Donghai WANG ${ }^{1}$, \\ Gang $\mathrm{LI}^{1}$, Wandong SU ${ }^{1}$ \\ 1Shandong University, Qilu Hospital, Department of Neurosurgery, Jinan, China \\ ${ }^{2}$ Caoxian People Hospital, Department of Neurosurgery, Heze, China
}

\section{ABSTRACT}

AIM: To assess the association between inflammatory response and early brain injury (EBI), and the association between inflammatory response and the following pneumonia after aneurysmal subarachnoid hemorrhage (SAH).

MATERIAL and METHODS: Eighty-nine patients with spontaneous SAH and 12 patients with unruptured aneurysm were included in this prospective study. The systemic inflammatory biomarkers such as C-reactive protein (CRP), IL-1ß, IL-2,IL-6,IL-8, IL10 and $\mathrm{T}$ leukocyte subsets were measured within 24 hours after admission. Their clinical features and laboratory findings were clearly reviewed and univariate analysis was used to find the main predictors.

RESULTS: The levels of serum inflammatory cytokines especially IL-6 $(p=0.004)$ and CRP $(p=0.014)$ would significantly increase after aneurysm SAH. Higher Fisher grades on admission result in higher levels of IL-6 and IL-10 (pIL-6=0.003. plL-10=0.002), and higher levels of IL-6, IL-10 and CRP were significantly associated with severe EBI, and increased the susceptibility to pneumonia $(p<0.05)$. The counts of CD3+ T Cells would decrease after aneurysm rupture $(p=0.001)$, especially in patients with a poor initial clinical grade. A reversed correlation between IL-6 level and CD3 T cells count was revealed in this study $(p=0.014, r=-0.249) ;$ a lower CD4 T-Cells counts might lead to subsequent pneumonia after SAH $(p=0.041)$. The levels of serum inflammatory cytokines were not different between aneurysmal and non-aneurysmal SAH.

CONCLUSION: Systemic inflammatory response would be activated after aneurysm rupture; a similar systemic inflammatory response would be noticed in non-aneurysmal SAH. The degree of inflammatory response could reflect the severity of EBI, and excessive inflammatory response could also aggravate EBI, induce immunodepression and increase the susceptibility to infections. Inflammatory cytokines such as IL-6, IL-10 and CRP are important predictors.

KEYWORDS: Subarachnoid hemorrhage, Early brain injury, Inflammatory response, Cytokines, Immunodepression

\section{INTRODUCTION}

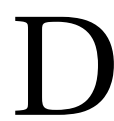
espite early surgical or endovascular treatment, aneurysmal subarachnoid hemorrhage (SAH) still has a high mortality and morbidity rate. Recently, early brain injury (EBI) was considered as an important determinant factor of outcome except delayed cerebral vasospasm (DCV) after aneurysmal SAH $(6,19)$. The exact mechanism of EBI has still not been clearly demonstrated and the levels of inflammatory cytokines such as interleukin-6 (IL-6) and C-reactive protein (CRP) would increase early after $\mathrm{SAH}$, inflammatory response would have been involved in the course of EBI. So far, most recent studies on inflammatory cytokines in $\mathrm{SAH}$ have focused on DCV while fewer studies have focused on EBI and its complications such as pneumonia, which influence the clinical course of aneurysmal SAH. Clinically, a serious initial clinical grade usually indicates a severe EBI after SAH (15), and infection is usually associated with immune dysfunction. 
The concentration of inflammatory cytokines could reflect the degree of inflammatory response. In this prospective study, the initial concentrations of systemic inflammatory biomarkers were measured to assess the association between inflammatory response and the severity of EBI, and the association between inflammatory response and the complication of pneumonia after aneurysmal SAH.

\section{MATERIAL and METHODS}

A series of patients with spontaneous SAH treated in our hospital from May 2012 to August 2013 were prospectively enrolled in this study. This study was approved by the ethical board of Qilu Hospital, Shandong University. All enrolled patients were admitted within 24 hours of symptom onset, and their hemorrhages were confirmed by head computerized tomography (CT) scan performed within 24 hours of the first symptoms. Exclusion criteria included patients with $\mathrm{SAH}$ caused by trauma, arteriovenous malformation and other structural lesions; patients with a history of infectious disease, surgery or major trauma in the previous month; patients with administration of anti-inflammatory or antibiotic drugs, or corticosteroids in the previous month; patients with concurrent major renal, hepatic and cancerous disease, or with obvious clinical evidence of in-hospital acquired infections before fasting blood sample tests. All SAH patients underwent standard monitoring and received the same medical treatment for aneurysmal SAH. The patients' neurological and general medical condition, the demographic, clinical and radiological features, laboratory findings and outcomes were clearly reviewed. The initial neurological findings were evaluated using the Hunt and Hess grading system $(\mathrm{H} \& \mathrm{H})$, and the patients were divided into two groups (mild: $\mathrm{H} \& \mathrm{H} 1-3$; poor: $\mathrm{H} \& \mathrm{H}$ 4-5). The amount of subarachnoid hemorrhage was classified using the modified Fisher scale (MFS) (5). DCV was defined as a focal neurological deficit or deterioration of consciousness occurring 3 to 14 days after the onset of $\mathrm{SAH}$, which was either confirmed of infarction on CT scan or could not be explained by other possible causes, such as a surgical procedure, re-bleeding, acute hydrocephalus, intracerebral hemorrhage, infections, seizures or electrolyte disturbances. Diagnosis of pneumonia was based on the following criteria: fever $\left(>38^{\circ} \mathrm{C}\right)$; abnormal respiratory examination; pulmonary infiltrates on chest $\mathrm{x}$-rays or CT scan; productive cough with purulent sputum; positive microbiological cultures from the lower respiratory tract or blood cultures. The patients were followed up via the outpatient clinic or by telephone interview 6 months after discharge and the outcome was assessed according to the Glasgow Outcome Scale (GOS) and was divided into two groups (GOS 4-5 was defined as 'favorable outcome', GOS 1-3 as 'poor outcome').

All fasting blood samples were obtained between 7:00 AM and 8:00 AM within 24 hours after admission; the blood was drawn and immediately sent to the laboratory. The analyses were performed according to the laboratory standards and the internal quality control protocol. The blood tests included total white blood cell (WBC) count, triglycerides (TG), cholesterol (CHOL), low-density lipoprotein (LDL), high-density lipoprotein
(HDL), inflammatory cytokines such as CRP, IL-1 $1 \beta, I L-2, I L-6$, IL-8 and IL-10, and the counts of T leukocyte subsets. The limits detection of these respective assays were $0.25 \mathrm{pg} / \mathrm{ml}$ for CRP, $4.5 \mathrm{pg} / \mathrm{ml}$ for IL-1 $13,0.5 \mathrm{pg} / \mathrm{ml}$ for IL-2, $0.2 \mathrm{pg} / \mathrm{ml}$ for IL-6, $0.5 \mathrm{pg} / \mathrm{ml}$ for IL-8, and $4.5 \mathrm{pg} / \mathrm{ml}$ for IL-10.The counts of leukocyte subsets (CD4+/CD8+ T) were determined by fluorescent-activated cell sorter. Data were analyzed using SPSS software. Appropriate statistical tests including Fisher's exact test, Chi-squared (x2) test or Student's t-test were used to find the significant factors related to the initial clinical grade, outcome and the complications such as DCV and infections. Values of $p<0.05$ were taken as statistically significant, and $p$ values between 0.05 and 0.10 were considered to be a trend. Spearson correlation was used to reveal the relationship between inflammatory cytokines.

\section{RESULTS}

Eighty-nine patients were initially included in this prospective study, of whom 78 patients presented with aneurysmal SAH and 11 patients presented with non-aneurysmal SAH. Another 12 patients with unruptured aneurysm who met the exclusion criteria were also included in this prospective study to make a total of 101 patients.

The basic clinical characteristics of these included patients are demonstrated in Table I. There were 70 (69.4\%) female and $31(30.6 \%)$ males, with ages ranging from 23 to 74 years (average: 53 years). The sex and age distributions were similar in each group. $39(38.6 \%)$ patients had a history of hypertension, 19 (18.8\%) smoking, 17 (16.8\%) drinking and $5(5 \%)$ diabetes, the distribution of these clinical risky factors were similar in each group. The serum lipids examination including TG, CHOL, LDL and HDL also had a similar distribution in each group.

The H\&H grade was 1-3 in 79 patients with SAH and 4-5 in 10 patients with $\mathrm{SAH}$; the Fisher grade was $1-2$ in 41 patients with SAH and 3-4 in 48 patients with SAH (Table II). The initial clinical grade and the amount of hemorrhage had a similar distribution in patients with aneurysmal SAH and patients with non-aneurysmal SAH.

In this study, 113 aneurysms were finally revealed in 90 patients (including 78 with aneurysmal SAH and 12 with unruptured aneurysm), 65 of whom underwent surgical treatment. Fifteen underwent endovascular treatment and 10 were treated conservatively. There was no obvious difference in the location, number and methods of treatment in patients with ruptured and un-ruptured aneurysm.

DCV were noticed in 22/89 patients with SAH (Table II), 21 of whom occurred in patients with aneurysmal SAH. The initial clinical condition, the amounts of SAH and the presence of ventricular hemorrhage were the major clinical factors leading to DCV in patients with aneurysmal SAH $(p<0.05)$. Pneumonia was noticed in $14 / 78$ patients with aneurysmal SAH. The common clinical factors such as the initial clinical condition, the amount of $\mathrm{SAH}$, the presence of ventricular hemorrhage and DCV were significantly associated with pneumonia in patients with aneurymal SAH $(p<0.05)$. At the last follow- 
up, $64(82 \%)$ patients with aneurysmal SAH had a favorable outcome (GOS 4-5), and 18\% (14) had a poor outcome (GOS 1-3). The clinical factors such as the initial clinical condition, the amount of SAH , the presence of ventricular hemorrhage, $\mathrm{DCV}$ as well as re-bleeding were significantly associated with the prognosis $(p<0.05)$.

The levels of inflammatory markers in patients with aneurysm are shown in Table III. The levels of inflammatory cytokines and especially IL-6 $(p=0.004)$ and CRP $(p=0.014)$ would increase after aneurysm rupture. However, the difference was not significant for the levels of IL-1 $\beta$, IL-2, IL-8 and IL-10 after aneurysm rupturing.

In patients with aneurymal $\mathrm{SAH}$, the levels of inflammatory markers were significantly associated with the initial neurological condition (Table IV), and were higher in patients with

Table I: The Basic Clinical Features of the Patients in this Study a poor clinical grade $(\mathrm{H} \& \mathrm{H}: 4-5)$. The patients with $\mathrm{H} \& \mathrm{H}$ of $4-5$ on admission had much higher levels of serum IL-6 , IL-10 and CRP than patients with a mild clinical grade $(\mathrm{H} \& \mathrm{H}: 1-3)$ $\left(p_{\mathrm{IL}-6}=0.015 . p_{\mathrm{IL}-10}=0.009 \cdot \mathrm{p}_{\mathrm{CRP}}=0.018\right)$. However, there were no differences in levels of IL-1 $\beta$, IL-2 and IL-8 between them. Similarly, patients with higher Fisher grades on admission showed more prominent increases in the levels of IL-6 and IL-10 $\left(p_{\mathrm{IL}-6}=0.003 . \mathrm{p}_{\mathrm{IL}-10}=0.002\right)$, but not in the levels of IL-1 $\beta$, IL-2, IL-8 and CRP. Higher levels of inflammatory cytokine such as IL-6, IL-10 and CPR could predict pneumonia (Table V). The levels of IL-6, IL-10 and CPR were much higher in patients with subsequent pneumonia $\left(p_{\mathrm{IL}-6}=0.001 . p_{\mathrm{IL}-10}=0.003\right.$. $\left.p_{\mathrm{CRP}}=0.002\right)$; a similar trend was noticed in levels of IL-2 $(p=0.077)$. There were no difference in levels of IL-1 $\beta$ and IL- 8 between patients with infections and patients without infections. In this study, higher levels of IL-6, IL-10 and CRP could lead to DCV and a poor outcome in patients with aneurysmal SAH.

\begin{tabular}{|c|c|c|c|c|}
\hline & $\begin{array}{l}\text { Aneurysmal SAH } \\
(n=78)\end{array}$ & $\begin{array}{c}\text { Non-aneurysmal SAH } \\
(n=11)\end{array}$ & $\begin{array}{l}\text { Unruptured aneurysm } \\
\qquad(n=12)\end{array}$ & $\begin{array}{c}\text { Total } \\
(n=101)\end{array}$ \\
\hline Age (y) (mean range) & $53(23-74)$ & $52(34-68)$ & $53(44-74)$ & $53(23-74)$ \\
\hline Current smoking & 15 & 2 & 2 & 19 \\
\hline Diabetes & 4 & 0 & 1 & 5 \\
\hline $\mathrm{TG}(\mathrm{mmol} / \mathrm{L})$ & $1.32 \pm 0.67$ & $1.11 \pm 0.61$ & $1.51 \pm 0.31$ & $1.34 \pm 0.73$ \\
\hline $\mathrm{CHOL}(\mathrm{mmol} / \mathrm{L})$ & $4.41 \pm 0.99$ & $4.22 \pm 0.63$ & $4.64 \pm 0.57$ & $4.42 \pm 0.91$ \\
\hline
\end{tabular}

SAH: Subarachnoid hemorrhage, y: Years, TG: Triglyceride, CHOL: Cholesterol, LDL: Low-density lipoprotein, HDL: High-density lipoprotein.

Table II: The clinical features of the patients with SAH $(n=89)$.

\begin{tabular}{|c|c|c|c|}
\hline & & Aneurysmal SAH $(n=78)$ & Non-aneurysmal SAH $(n=11)$ \\
\hline \multirow{2}{*}{$\mathrm{H} \& \mathrm{H}$ grade } & $1-3$ & 68 & 11 \\
\hline & $4-5$ & 10 & 0 \\
\hline \multirow{2}{*}{ MFS } & $1-2$ & 33 & 8 \\
\hline & $3-4$ & 45 & 3 \\
\hline \multirow{2}{*}{ DVC } & No & 57 & 10 \\
\hline & Yes & 21 & 1 \\
\hline \multirow{2}{*}{ Pneumonia } & No & 64 & 11 \\
\hline & Yes & 14 & 0 \\
\hline \multirow{2}{*}{ Outcome } & Favorable & 64 & 10 \\
\hline & Unfavorable & 14 & 1 \\
\hline
\end{tabular}

H\&H: Hunt and Hess, MFS: Modified Fisher scale, DVC: Delayed cerebral vasospasm, SAH: Subarachnoid hemorrhage. 
Table III: The Systemic Inflammatory Markers and T Leukocyte Subsets in Different Groups

\begin{tabular}{|c|c|c|c|c|}
\hline & & Aneurysmal SAH $(n=78)$ & Non-aneurysmal SAH $(n=11)$ & Unruptured aneurysm $(n=12)$ \\
\hline WBC & & $10.73 \pm 4.31$ & $9.10 \pm 3.72$ & $7.14 \pm 3.01$ \\
\hline \multirow{2}{*}{$\mathrm{IL}-1 \beta$} & $<45$ & 69 & 11 & 10 \\
\hline & $\geq 4.5$ & 9 & 0 & 2 \\
\hline IL-2 (pg/ml) & & $445.97 \pm 233.72$ & $411.63 \pm 253.44$ & $363.08 \pm 169.21$ \\
\hline $\mathrm{IL}-6^{\star}(\mathrm{pg} / \mathrm{ml})$ & & $44.42 \pm 47.28$ & $33.71 \pm 65.07$ & $4.41 \pm 3.92$ \\
\hline IL-8 (pg/ml) & & $73.899 \pm 94.67$ & $46.88 \pm 41.07$ & $38.55 \pm 30.20$ \\
\hline \multirow{2}{*}{ LI-10 } & $<4.5$ & 60 & 10 & 10 \\
\hline & $\geq 4.5$ & 18 & 1 & 2 \\
\hline $\mathrm{CRP}^{*}(\mathrm{pg} / \mathrm{ml})$ & & $19.12 \pm 35.99$ & $15.10 \pm 30.05$ & $8.53 \pm 15.88$ \\
\hline $\mathrm{CD}^{+*}$ & & $61.19 \pm 10.86$ & $58.57 \pm 11.29$ & $68.25 \pm 2.59$ \\
\hline $\mathrm{CD}^{+}$ & & $35.80 \pm 9.20$ & $34.77 \pm 6.70$ & $40.37 \pm 6.26$ \\
\hline $\mathrm{CD8}^{+}$ & & $21.64 \pm 6.97$ & $20.99 \pm 8.24$ & $24.03 \pm 5.72$ \\
\hline $\mathrm{CD}^{+} / \mathrm{CD}^{+}$ & & $1.89 \pm 0.94$ & $2,04 \pm 1.20$ & $1.82 \pm 0.67$ \\
\hline
\end{tabular}

*indicates a significant difference between aneurysmal SAH and unruptured aneurysm, with a $p$ value $<0.05$.

SAH: Subarachnoid hemorrhage, WBC: White blood cell.

Table IV: The Systemic Inflammatory Markers and T Leukocyte Subsets in Aneurysmal SAH ( $\mathrm{n}=78)$

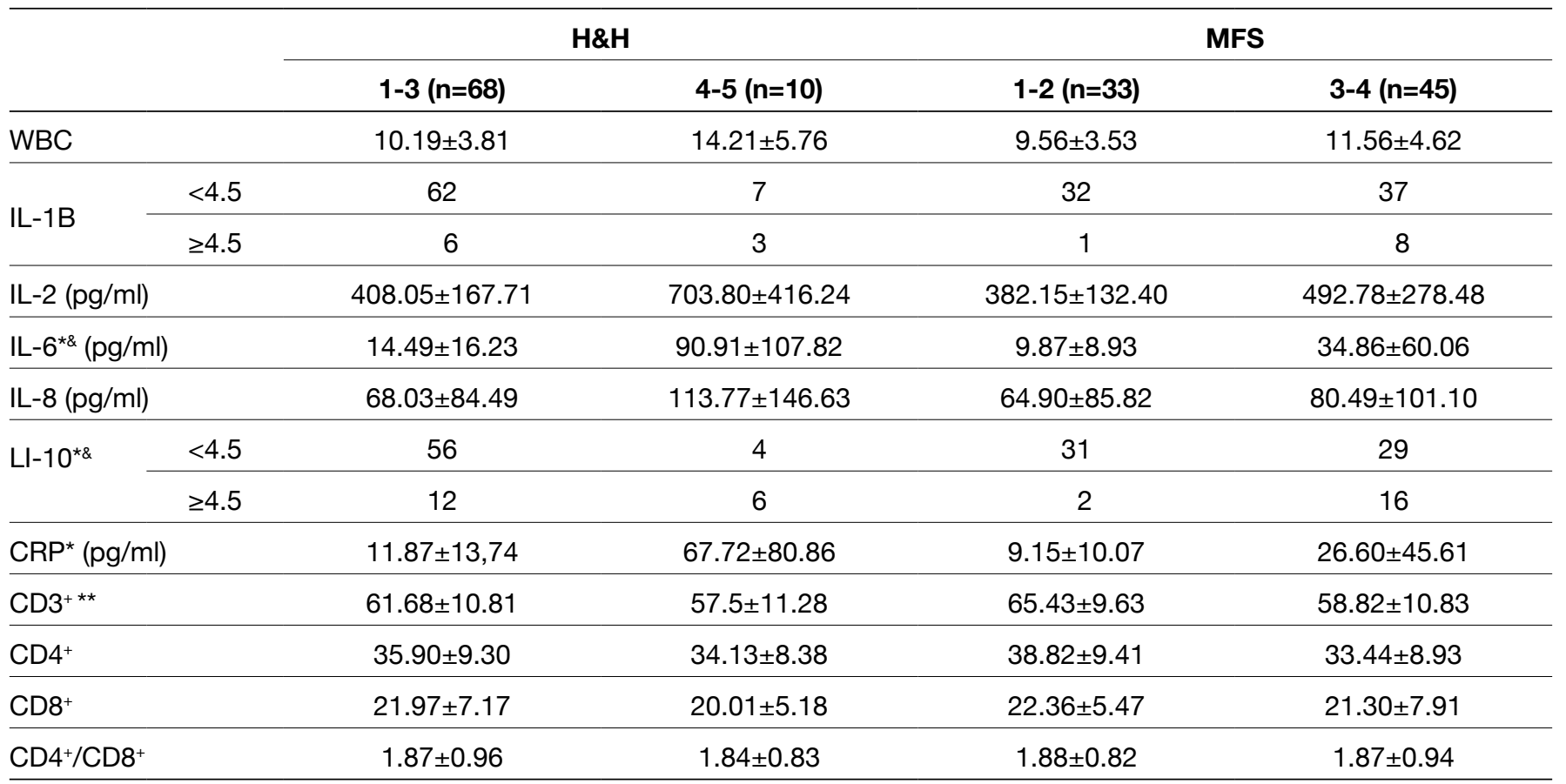

*indicates a significant difference between patients with $H \& H$ grade of 1-3 and grade of 4-5, with a $p$ value $<0.05$;

**indicates a difference between patients with H\&H grade of 1-3 and grade of 4-5, with a p value between 0.05 and 0.1

${ }^{*}$ indicates a significant difference between patients with MFD grade of 1-2 and grade of 2-4, with a $p$ value $<0.05$

SAH: Subarachnoid hemorrhage, WBC: White blood cell, MFS: Modified Fisher scale, H\&H: Hunt and Hess. 
Table V: The Association between Systemic Inflammatory Markers and the Complication and Outcome of Patients with Aneurysmal $\mathrm{SAH}(\mathrm{n}=78)$

\begin{tabular}{|c|c|c|c|c|c|c|c|}
\hline & & \multicolumn{2}{|c|}{ Pneumonia } & \multicolumn{2}{|c|}{ Vasospasm } & \multicolumn{2}{|c|}{ Outcome } \\
\hline $\begin{array}{l}\text { IL-1B } \\
\text { (pg/ml) }\end{array}$ & $<4.5$ & 58 & 11 & 51 & 18 & 57 & 12 \\
\hline $\mathrm{IL}-6^{\star \& \$}(\mathrm{pg} / \mathrm{ml})$ & & $13.68 \pm 16.00$ & $73,19 \pm 94.71$ & $14.96 \pm 16.92$ & $51.62 \pm 84.91$ & $17.79 \pm 28.94$ & $57.42 \pm 92.73$ \\
\hline IL-8 (pg/ml) & & $70.80 \pm 88.15$ & $92.80 \pm 123.37$ & $75.69 \pm 91.14$ & $72.27 \pm 107.57$ & $69.54 \pm 86.58$ & $100.88 \pm 129.88$ \\
\hline Ll-10*\$(pg/ml) & $<4.5$ & 54 & 3 & 47 & 13 & 53 & 7 \\
\hline $\mathrm{CD}^{+*}{ }^{*}$ & & $36.95 \pm 8.96$ & $31.39 \pm 9.27$ & $36.11 \pm 9.27$ & $34.94 \pm 9.18$ & $36.59 \pm 9.08$ & $32.11 \pm 9.19$ \\
\hline $\mathrm{CD8}^{+}$ & & $21.61 \pm 6.68$ & $21.75 \pm 8.25$ & $21.76 \pm 6.85$ & $21.30 \pm 7.47$ & $21.60 \pm 6.73$ & $21.80 \pm 8.28$ \\
\hline $\mathrm{CD}^{+}{ }^{+} / \mathrm{CD}^{+}+$ & & $1.94 \pm 0.96$ & $1.65 \pm 0.85$ & $1.89 \pm 0.95$ & $1.87 \pm 0.95$ & $1.92 \pm 0.93$ & $1.70 \pm 1.00$ \\
\hline
\end{tabular}

*indicates a significant difference between patients with infections and patients without non-infections, with a $p$ value $<0.05$

$\&$ indicates a significant difference between patients with vasospasm and patients without vasospasm, with a $p$ value $<0.05$

$\$$ indicates a significant difference between patients with a good outcome and patients with poor outcome, with a $p$ value $<0.05$

SAH: Subarachnoid hemorrhage, WBC: White blood cell.

The counts of leukocytes and their subsets (CD4+/CD8+ T) were also studied in this study. The counts of CD3+ T Cells would decrease significantly after aneurysm rupture $(p=0.001)$, and the count of CD3+ T Cells was associated with the initial neurological condition and tended to be lower in patients with a poor clinical grade, though the difference did not reach statistical significance $(p=0.056)$. There were no differences in the counts of subsets $(C D 4+/ C D 8+T)$. A reversed correlation between IL- 6 levels and CD3+ T cells count was revealed $(p=0.014, r=-0.249)$ in this study. Meanwhile, the cell immune response was significantly associated with subsequent infections, the counts of CD3+ T Cells, especially CD4+ $\mathrm{T}$-Cells were much lower in patients with pneumonia $(p=0.043$, $\mathrm{p}=0.041$ ).

In this study, a similar system inflammatory response was triggered after $\mathrm{SAH}$, whether aneurysmal or non-aneurymal $\mathrm{SAH}$. There was no difference in the levels of the above inflammatory cytokines between patients with aneurysmal and patients with un-aneurysmal SAH. The cell immune response was similar in patients with $\mathrm{SAH}$, whether aneurysmal or un-aneurysmal. There was no difference in the counts of T-lymphocyte subtype including CD4+, CD8+ T cell in patients with aneurysmal and non-aneurysmal SAH.

\section{DISCUSSION}

$\mathrm{EBI}$ is described as immediate injury to brain within 72 hours after SAH (6). It is an important determinant factor of outcome after aneurysmal SAH (19). Direct mechanical insults and the hypoperfusion caused by elevated intracranial pressure could induce EBI $(6,19)$, and inflammatory response could also aggravate EBI. The ectopic blood cells and breakdown products will induce leukocyte aggregation and endothelial cell and microglia activation, which will secrete inflammatory cytokines and cause a local inflammatory response. Although appropriate local inflammatory response could accelerate bleeding absorption and brain function recovery, an excessive inflammatory response would be harmful to the brain as it could induce release of inflammatory and apoptotic factors, vasoconstrictors, reactive oxygen species, cytotoxic proteases and so on which will induce cerebral vasospasm, blood-brain barrier injury, brain edema, neurocyte apoptosis and necrosis, and all these would aggravate early brain injury $(2,19)$. Furthermore, acute insult would induce a systemic inflammatory cascade reaction, which would further aggravate metabolic dysfunctions and brain tissue damage. Excessive inflammatory responses therefore play a critical role in EBI, and a high concentration of inflammatory cytokines could aggravate EBI. In one sense, the degree of inflammatory responses could reflect the severity of EBI. Similar to our study, other studies also revealed that high levels of inflammatory cytokines such as IL-2, IL-6, IL-8 and CRP correlated with a higher clinical grade $(4,9,11,17)$. The process of antiinflammation would be activated simultaneously after $\mathrm{SAH}$, 
and the levels of IL-10 would increase after SAH, especially in patients with a poor clinical grade in this study; it may provide a negative feedback to limit the harm of the pro-inflammatory response. However, there may be an unlimited and abnormal inflammatory response in patients with a poor clinical grade. The degree of pro-inflammatory and anti-inflammatory responses might be not proportionate, which would aggregate $\mathrm{EBI}$ and result in a poor clinical grade.

Not only is it possible for the local inflammatory cytokines to be passively released to the plasma through the disrupted blood-brain barrier after SAH (12), the peripheral target cells could also initiatively secrete inflammatory cytokines. In this study, a higher Fisher grade on CT scan admission indicated higher levels of inflammatory cytokines. Much more hemorrhage indicates much more secretion of local inflammatory cytokines, which could then directly activate the sympathetic nervous system (SNS) (22); secondly, considerable hemorrhage and increased intracranial pressure could compress the brain stem, hypothalamus and spinal cord, which would immediately activate SNS. The latter would stimulate peripheral targets secreting inflammatory cytokines such as IL-6 and IL-8 $(1,7)$. Meanwhile, the activated SNS would persist at least one week after SAH (14), which would lead to permanent high inflammatory cytokine levels. Although one study found that patients with non-aneurysmal SAH had a milder inflammatory response than patients with aneurysmal SAH (13), the intensity of systemic inflammation response in our study was similar in patients with aneurysmal and nonaneurysmal $\mathrm{SAH}$, which indicates a similar mechanism of generation and action.

High levels of systemic inflammatory cytokines such as IL2, IL-6 and CRP have been demonstrated to predict future systemic infections after stroke $(3,23)$. A similar result was also revealed in aneurysmal SAH in this study. Clinically, the patients with intense inflammatory response often had a poor clinical grade, were usually unconscious, and required long-term bedridden or mechanical ventilation, and all of these would increase the susceptibility of infections. The proinflammatory cytokines could activate the SNS and induce catecholamine release, and the latter could directly suppress Th1 activities and cellular immune response (16). Furthermore, catecholamines could also induce interleukin-10 release from monocytes (20). Higher serum IL-10 levels on admission would increase the susceptibility of subsequent clinical infections in this study. The plasma IL-10 levels were still higher in patients who developed infections, even the patients treated with anti-infective drugs (10). As an anti-inflammatory cytokine, IL-10 has pleiotropic effects: it could not only limit excessive inflammatory response, but also impair $\mathrm{T} 1$ helper cells and monocyte functions and induce immunosuppresion (8). The patients with subsequent infections presented a marked loss of lymphocytes and especially CD4+ subsets in our study, and similar results were also revealed in another study (18). A reversed correlation between IL-6 level and CD3 T cells count was also demonstrated in this study. Furthermore, the pro-inflammatory cytokines could activate the hypothalamuspituitary cortex axis and induce glucocorticoid secretion, and lead to immunosuppression (21). An intense pro-inflammatory response might therefore impair T1 helper cells through multiple mechanisms and the impaired cellular immune would induce immunodepression and increase the susceptibility to clinical infections after SAH.

There is a close relationship between these inflammatory cytokines: they may have a common origin or generating condition, or belong to a complicated and closely connected inflammatory network. One thing one must keep in mind is that despite minor changes in some inflammatory cytokines, those cytokines may have also been involved in the pathological process of aneurysmal SAH. Their effects may be limited or a slight increase would induce pathological processes; secondly, most recent studies focus on the disadvantage of inflammatory response but as a common phenomenon, inflammatory response is beneficial to weaken exogenous and endogenous insults and plays an important part in the processes of tissue remodeling and regeneration. These mechanism should be also further investigated.

There are some limitations in this study: the levels of inflammatory markers were only measured once and intraindividual variation and dynamic changes in these markers should be taken into account. The sample of cerebrospinal fluid (CSF) could not be obtained safely before surgical intervention, so the levels of inflammatory markers in CSF were not measured, and the cerebral local inflammatory response could not be fully evaluated by hematological indexes.

\section{- CONCLUSION}

Systemic inflammatory response would be activated after aneurysm rupture; a similar systemic inflammatory response would be noticed in non-aneurysmal SAH. The degree of inflammatory response could reflect the severity of EBI. Excessive inflammatory response could also aggravate EBI, induce immunodepression and increase the susceptibility of infections. Inflammatory cytokines such as IL-6, IL-10 and CRP are important predictors.

\section{- REFERENCES}

1. Catania A, Lonati C, Sordi A, Gatti S: Detrimental consequences of brain injury on peripheral cells. Brain Behav Immun 23(7):877-884, 2009

2. Dénes $A$, Ferenczi $S$, Kovács KJ: Systemic inflammatory challenges compromise survival after experimental stroke via augmenting brain inflammation, blood-brain barrier damage and brain oedema independently of infarct size. $J$ Neuroinflammation 8:164, 2011

3. Fluri F, Morgenthaler NG, Mueller B, Christ-Crain M, Katan M: Copeptin, procalcitonin and routine inflammatory markerspredictors of infection after stroke. PLoS One 7(10):e48309, 2012

4. Fountas KN, Tasiou A, Kapsalaki EZ, Paterakis KN, Grigorian AA, Lee GP, Robinson JS Jr: Serum and cerebrospinal fluid C-reactive protein levels as predictors of vasospasm in aneurysmal subarachnoid hemorrhage. Neurosurg Focus 26(5):E22, 2009 
5. Frontera JA, Claassen J, Schmidt JM, Wartenberg KE, Temes $\mathrm{R}$, Connolly ES Jr, MacDonald RL, Mayer SA: Prediction of symptomatic vasospasm after subarachnoid hemorrhage: The modified Fisher scale. Neurosurgery 59(1):21-27, 2006

6. Fujii M, Yan J, Rolland WB, Soejima Y, Caner B, Zhang $\mathrm{JH}$ : Early brain injury, an evolving frontier in subarachnoid hemorrhage research. Transl Stroke Res 4(4):432-446, 2013

7. Gao C1, Liu X, Shi H, Xu S, Ji Z, Wang C, Wu P, Liu Z, Zhao $S:$ Relationship between sympathetic nervous activity and inflammatory response after subarachnoid hemorrhage in a perforating canine model. Auton Neurosci 147(1-2):70-74, 2009

8. Grütz G: New insights into the molecular mechanism of interleukin-10-mediated immunosuppression. J Leukoc Biol 77(1):3-15, 2005

9. Hanafy KA, Morgan Stuart R, Fernandez L, Schmidt JM, Claassen J, Lee K, Sander Connolly E, Mayer SA, Badjatia N: Cerebral inflammatory response and predictors of admission clinical grade after aneurysmal subarachnoid hemorrhage. J Clin Neurosci 17(1):22-25, 2010

10. Klehmet J, Harms H, Richter M, Prass K, Volk HD, Dirnagl $U$, Meisel A, Meisel C: Stroke-induced immunodepression and post-stroke infections: Lessons from the preventive antibacterial therapy in stroke trial. Neuroscience 158(3):11841193, 2009

11. Kwon KY, Jeon BC: Cytokine levels in cerebrospinal fluid and delayed ischemic deficits in patients with aneurysmal subarachnoid hemorrhage. J Korean Med Sci 16(6):774-780, 2001

12. Morganti-Kossmann MC, Satgunaseelan L, Bye N, Kossmann T: Modulation of immune response by head injury. Injury 38(12):1392-1400, 2007

13. Muroi C, Bellut D, Coluccia D, Mink S, Fujioka M, Keller E: Systemic interleukin-6 concentrations in patients with perimesencephalic non-aneurysmal subarachnoid hemorrhage. J Clin Neurosci 18(12):1626-1629, 2011
14. Naredi S, Lambert G, Edén E, Zäll S, Runnerstam M, Rydenhag $B$, Friberg P: Increased sympathetic nervous activity in patients with nontraumatic subarachnoid hemorrhage. Stroke 31(4):901-906, 2000

15. Nishizawa S: The roles of early brain injury in cerebral vasospasm following subarachnoid hemorrhage: From clinical and scientific aspects. Acta Neurochir Suppl 115:207-211, 2013

16. Prass K, Meisel C, Höflich C, Braun J, Halle E, Wolf T, Ruscher K, Victorov IV, Priller J, Dirnagl U, Volk HD, Meisel A: Strokeinduced immunodeficiency promotes spontaneous bacterial infections and is mediated by sympathetic activation reversal by poststroke $\mathrm{T}$ helper cell type 1-like immunostimulation. $\mathrm{J}$ Exp Med 198(5):725-736, 2003

17. Sarrafzadeh A, Schlenk F, Gericke C, Vajkoczy P: Relevance of cerebral interleukin-6 after aneurysmal subarachnoid hemorrhage. Neurocrit Care 13(3):339-346, 2010

18. Sarrafzadeh A, Schlenk F, Meisel A, Dreier J, Vajkoczy P, Meisel C: Immunodepression after aneurysmal subarachnoid hemorrhage. Stroke 42(1):53-58, 2011

19. Sehba FA, Hou J, Pluta RM, Zhang JH: The importance of early brain injury after subarachnoid hemorrhage. Prog Neurobiol 97(1):14-37, 2012

20. Woiciechowsky C, Asadullah K, Nestler D, Eberhardt B, Platzer C, Schöning B, Glöckner F, Lanksch WR, Volk HD, Döcke WD: Sympathetic activation triggers systemic interleukin-10 release in immunodepression induced by brain injury. Nat Med 4(7):808-813, 1998

21. Woiciechowsky C, Schöning B, Lanksch WR, Volk HD, Döcke WD: Mechanisms of brain-mediated systemic antiinflammatory syndrome causing immunodepression. J Mol Med (Berl) 77(11):769-780, 1999

22. Woiciechowsky C, Schöning B, Lanksch WR, Volk HD, Döcke WD: Catecholamine-induced interleukin-10 release: $A$ key mechanism in systemic immunodepression after brain injury. Crit Care 3(6):R107-R111, 1999

23. Zhang $\mathrm{H}$, Li X: Correlation between inflammatory factors and post-stroke pneumonia in diabetic patients. Exp Ther Med 6 (1):105-108, 2013 